who was the members of reputed IBM group and at that time Ted Codd was a computer scientist at IBM's San Jose Research Laboratory who was proposing a new way of organization for data that he called the "relational data model" for the persistent data. He named This Query Language SEQUEL (Structured English QUEry Language).In 1976-77 next reluctant version was quickly named SEQUEL/2, but had to be renamed to $S Q L$ due to legal reasons[5].

In the era of mainframes and business applications many developers use a mature query technology that is SQL. From the beginning RDBMS were very popular. In fact, in 1979 the first commercial implementation was released by a small and unknown software company named "Relational Software". This unknown software company now becomes a popular as Oracle. Johannes Zollmann described in his chapter about the ACID properties of the RDBMS. He also notes that ACID properties give strong guarantees on consistency [9].

Though ACID makes a strong to relational databases some drawback of type is, These databases were works with single server that means way of enhancing the capacity is only upgrading the server. Another researcher Antro Salminen note that in his seminar that for scale-up the RDBMS only way is by adding the Hardware processing power[6] RDBMS also has physical storage limit[3].Hence Such database was required that support current generation web, mobile, and other applications to operate at any scale.As need is a Mother of innovations, when everyone was facing a problem of Big data there was a introduction of NoSQL by the researchers.

NoSQL is divided into 4 different types as Key Values, Documents, Colum and Graphs. Each database has its own advantages. Selection of type is depending on the type of application and its requirement. Some of the advantage of NoSQL which make it powerful is Horizontal scaling, Demoralization, Replication.

NoSQL gives the benefit of time over relational databases' join queries by providing a feature of simple graph traversal operation queries. We can store frequently required information in one table in NoSQL with its horizontal Scaling property. NoSQL also able to handle all the table Joins at application level. Another feature of NoSQL is Data repetition is acceptable. This feature of NoSQL helps to improve efficiency and execution speed of query. However tables are not related in NoSQL so we need to very careful and needs to synchronize the data while updating the Tables.

Markus Winand wrote in his blog that Many NoSQL systems claimed to solve all performance problems by feature of horizontal scalability. Though for the current generation database scalability is limited to write operations and is accomplished with eventual consistency model. A strict consistency model of SQL databases make slows down write operations, still that does not necessarily imply bad throughput [19].

Migration of Data between SQL and NoSQL depending on the style of NoSQL database. Relational database expert Chris Bird observed syntactical difference NoSQL and SQL, Also found that hardness for migration like some mental gymnastics requirement for new users of NoSQL. Hence the process for migration is depend on which technology of NoSQL technology going to be selected for use. Some researchers had tried to cover the gap between RDB and GDB .Graph Databases are able to represent as graph of any kind of information, naturally accommodate changes in data[15]. Cypher Gremlin, are examples graph data query languages.

Some noSQL db doesn't support range queries or joins, users are restricted in what user can view and how fast user can view[10]. An Oracal white paper note that Choice of new system is an exciting, strategic business activity that usually entails working with new technologies, suppliers, and opportunities under the head of successful data migration in October 2011. It is also observed that without a sufficient knowledge of both source and target, data migration can cause major or minor or any hidden legacy problems, which is nothing but playing with risk. [17].

It is usually assumed that target of migration knows the existing systems and support a same structure. However NoSql Query Stucture is harder to gain user acceptance . Researcher Patrícia Cavoto observed in his research work that Complex Structer of relational DB cause the complexity can be while performing a data analysis in RDB. Also the relational models are not as flexible as the graph model for data analysis[11] .some researchers found that relational database might require very sophisticated and expensive operations also needs complex join operations to fetch required results in RDBMS which affect the performance and efficiency deterioration $[15,16]$.

In concern with rethinking and remodeling of data migration which is essential part of almost every organization, While the migration of data from SQL 
between NoSQL developers need to think about how to represent your existing model in new database[18] it means mechanism for storage and retrieval of data are designed in different way in both databases.

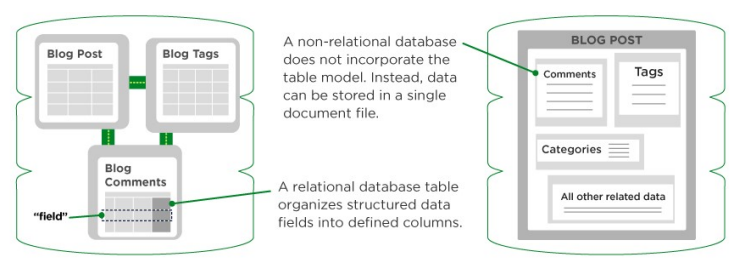

Figure 1: sql-vs-nosql-databases[21]

\section{Power of SQL:}

ACID compliancy: ACID property is the best power of SQL which reduces anomalies and protects the integrity database.

Security: In Handling of data in DBMSs security of data is important .In security concerns NoSQL databases loose his security by allowing permissions or access control in these systems are to be provided it means it lacks the with the respect to Security as compare to SQL.

\section{Limitations of SQL:}

Challenge of scalability and elasticity achievement is a huge related to the relational databases. Researcher also found that a small change to one table can cause changes across the system [20].It is observe that response time of an SQL query changes with many factors. One of them is volume and Scalability. These factors are also related to dependency of performance the data volume [19]

One another limitation of SQL is fixed set of columns which is not suitable for big data and hence there is a demand for non-relational databases. Leonardo Rocha and et ,al; point out that Challenges in Migration: Recontruction of Module

\section{Conclusion:}

From the survey we can conclude that -

a) existing softwares not only large and but also medium scale software applications are relye on rlational database.

b) Due to the variation in synatatical and storage structure of SQL and NoSQL thrir is problem of migration.

As amount of data stored in SQL has a impact on its performance. i.e Data stored and the performance are inversely proportional to each other. Effect of this is a query becomes slower. But can we improve this ratio by giving a power of Horizontal Scaling to SQL of NoSQL is the key questions when discussing dataset scalability.

\section{REFRENCES}

1. Computerworld "No to SQL" Anti-database movement gains steam. June 2009. http://www.computerworld.com/s/article/9135086/ No_to_SQL_Anti_database_movement_gains_steam.

2. J. Manyika, M. Chui, B. Brown, J. Bughin, R. Dobbs, C. Roxburgh, and A. H. Byers, "Big data: The next frontier for innovation, competition, and productivity," McKinsey Global Institute, pp

3. Couch DB - The Definitive Guide www.couchbase.com/ -binaries/content/assets/ website/ docs/ whitepapers

4. Donald D. Chamberlin, Editor: Craig Partridge "Early History of SQL" IEEE Annals of the History of Computing Published by the IEEE Computer Society 1058-6180/12/\$31.00 2012 IEEE

5. Frank Kalis, guest columnist, SQLServerCentral.com edited and annotated by Alan G. Labouseur "A Brief History of SQL"

6. Antro Salminen "Introduction to NoSQL" NoSQL Seminar2012@TUT

7. R. Cattell. Scalable sql and nosql data stores. SIGMOD Rec., 39(4):12-27, May 2011.

8. C. Strozzi. Nosql relational database management system. http://www.strozzi.it/cgi-bin/CSA/ tw7/I/en_US/NoSQL/HomePage, July 2012.

9. Johannes Zollmann "NoSQL Databases" Chapter 1 Typeset August 20, 2012

10. stackoverflow.com/questions/7782627/shiftingfrom-sql-to-nosql-and-to-which-db.

11. Patrícia Cavoto "ReGraph: Bridging Relational and Graph Databases". Thesis presented to the Institute of Computing of the University of Campinas,2016.

12. Patrícia Cavoto, Victor Cardoso, Régine VignesLebbe, and André Santanchè. Fish- Graph: A Network-Driven Data Analysis. In 11th IEEE International Conference on e-Science, e-Science 2015, Munich, Germany, August 31 - September 4, pages 177-186, 2015. 
13. Patrícia Cavoto and André Santanchè. AnnotationBased Method for Linking Local and Global Knowledge Graphs. In Proceedings of the Brazilian Seminar on Ontologies (ONTOBRAS 2015), São Paulo, Brazil, September 8-11, 2015.

14. Patrícia Cavoto and André Santanchè. ReGraph: Bridging Relational and Graph Databases. In Proceedings of the 30th Brazilian Symposium on Databases 2015 (SBBD, 2015), Demos and Applications Session, 2015.

15. Adrian Silvescu, "Graph Databases" Manpreet Kaur and Manpreet Singh "SQL2Neo : Moving Healthcare Data From Relational To Graph Databases" https://www.researchgate.net/publication/28312837 5 July 2015

16. Oracal White paper "Successful Data Migration" October 11 , oracal.com Copyright (C) 2011, Oracle and/or its affiliates.

17. Gwen Shapira "On the Difficulty of Data Migrations (Especially to NoSQL Databases) " blog Technical Track April 26, 2010.

18. Markus Winand "Response Time, Throughput and Horizontal Scalability" blogpost - use-the-indexluke A Gupde to database performance for developers.

19. Matt Allen "Relational Databases Are Not Designed For Scale".

20. Hiring Headquarters "SQL vs. NoSQL Databases: What's the Difference" blog.upwork.com

21. Aaron Schram and Kenneth M. Anderson. Mysql to nosql: Data modeling challenges in supporting scalability. In ACM SPLASH '12, pages 191-202, 2012.

22. Cansu Birgen "SQL vs NoSQL" theses-Norwegian University of Science and Technology.Dept of Chemical Engineering December 8, 2014 